\title{
Answer to Medical Quiz: Images
}

\begin{abstract}
Answer
Q1. (Fig. 1) CT angiogram of aorta and its branches showing kinking of proximal part of right common carotid artery. Right subclavian artery is visualized only in proximal part. Left subclavian artery stenosis and irregularities at its proximal part is noted. Right vertebral artery is not visualized. (Fig. 2) CT angiogram of abdominal aorta and its branches showing significant stenosis at proximal common iliac arteries on both sides and also at origin of both internal iliac arteries.
\end{abstract}

Q2. Takayasu arteritis.

\section{Review}

Takayasu arteritis (TA), also known as pulseless disease, occlusive thrombo-aortopathy, aortic arch syndrome or reverse coarctation, is a chronic large vessel systemic granulomatous vasculitis, primarily involving the aorta and its major branches. ${ }^{1}$ The disease was named for Mikito Takayasu, a Japanese Ophthalmologist, who presented the first case of TA in $1908 .{ }^{2}$

The typical age at onset of manifestation of TA is between 25 and 30 years with an 8:1 female preponderance. The criteria for making diagnosis of TA set by the American College of Rheumatology ${ }^{3}$ (ACR) and Sharma et al. ${ }^{4}$ have high sensitivity (91\% vs 96\%) and specificity ( $98 \%$ vs $96 \%$ ). TA can be divided into the following 6 types based on angiographic involvement: ${ }^{5}$ Type I - Branches of the aortic arch, Type IIa - Ascending aorta, aortic arch and its branches, Type IIb - Type IIa region plus thoracic descending aorta, Type III - Thoracic descending aorta, abdominal aorta, renal arteries or a combination, Type IV - Abdominal aorta, renal arteries or both, Type V - Entire aorta and its branches.
Vascular abnormalities in TA can be imaged by conventional angiography, magnetic resonance imaging (MRI), magnetic resonance angiogram (MRA), CT angiography or ultrasonography. Conventional angiography is the "gold standard" but invasive; can delineate the stenoses, occlusions and aneurysms and can be used for angioplasty. MRA is the preferred imaging method for follow up of patients. In our case, $\mathrm{CT}$ angiographic findings were consistent with TA.

Erythrocyte sedimentation rate (ESR) is elevated in $80 \%$ cases and C-reactive protein (CRP) in 50\% cases. Mild anemia is common. The white blood cell (WBC) count is usually normal or slightly elevated. The platelet count is elevated in one third cases with active disease. Our patient had high ESR and CRP. Corticosteroids e.g. prednisone, at a dose of 0.5 to $1 \mathrm{mg} / \mathrm{kg}$ per day is the cornerstone of treatment in active disease. Prophylactic treatment for osteoporosis and cardiovascular risk factors should be addressed properly. Surgery may be required in treating stenotic or aneurysmal lesions and aortic valve replacement.

\section{References}

1. Magge SN, Chen HI, Stiefel MF, Ernst L, Cahill AM, Hurst $\mathrm{R}$, et al. Multiple ruptured cerebral aneurysms in a child with Takayasu arteritis. J Neurosurg Pediatr 2008;1:83-87.

2. Takayasu M. A case of a peculiar change in the central retinal vessels. Acta Soc Ophthalmol Jpn 1908; 12:554.

3. Arend WP, Michel BA, Bloch DA, Hunder GG, Calabrese LH, Edworthy SM, et al. The American College of Rheumatology 1990 criteria for the classification of Takayasu arteritis. Arthritis Rheum 1990; 33:1129-34.

4. Sharma BK, Jain S, Suri S, Numano F. Diagnostic criteria for Takayasu arteritis. International J Cardiol 1996; 54 (suppl 2):141-47.

5. Hata A, Noda M, Moriwaki R, Numano F. Angiographic findings of Takayasu arteritis: new classification. Int J Cardiol 1996;54 Suppl:S155-63 